\title{
POLARONS BY THE NONLOCAL DYNAMICAL COHERENT POTENTIAL METHOD
}

\author{
S.V.IZVEKOV \\ T.Shevchenko Kyiv University, Department of Physics, \\ 6 Glushkov Av., UA-252022 Kyiv, Ukraine
}

Received February 26, 1997; Revised version September 16, 1997

The nonlocal dynamical coherent potential approximation (NDCPA) is formulated to calculate a single-electron (exciton) Green's function of polaron due to the interaction of an electron (exciton) with phonons with dispersion. This approximation is an extension of the dynamical CPA. The NDCPA provides an efficient means of calculating an approximate Green's function for a dynamical model of electrons (excitons) strongly coupled to optical or acoustical phonons, in the entire ranges of the electron (exciton)-phonon coupling strengths and electron (exciton) transfer. The electron (exciton)-phonon coupling in the Hamiltonian may involve terms of any order with respect to phonon operators. A set of recurrent equations is derived in the case of a system at zero temperatures, from which the coherent potential can be obtained as a function of energy $E$. The simple algorithm for the polaron spectra calculations is obtained for a linear electron (exciton)-phonon coupling in the antiadiabatic limit.

\section{Introduction}

Electron(exciton) with a lattice or molecular phonon cloud around it can be regarded as a new single particle state - respectively a lattice or molecular (exciton) polaron. At present, it is already almost impossible in a small space like this to present a trustworthy review of all the relevant work done in the field of polaron states up to now. Being one of the relatively simplest, most important and directly applicable examples of quasiparticle description of properties of many-particle systems, the polaron problem has served many times for both purely theoretical speculations and direct explanation of experimental data. The variety of aims corresponds to a variety of approaches as well as tools: from field-theoretical expansions in powers of electron(exciton)-phonon coupling constant [1], through different kinds of interpolation (variational) approaches [2] to small polaron theories invoking so-called polaron canonical transformation [3]. Each of the approaches is fitted to special purposes and is good in its own (broader or narrower) area of dimensionless parameter of the problem [3]. For instance, variational interpolation approaches are concentrated on the problem of the polaron dispersion law, leaving the polaron quasiparticle damping in the whole interval of parameters mostly aside. In the present work, it is attempted to partly fill this gap by submitting theory which is in principle able to give such information.

In order to determine a single-particle Green's function, which provides the information about the physical properties (energy spectrum, spectral functions) of polaron states, one should actually calculate a self-energy

(C) S.V.Izvekov, 1997

ISSN 0452-9910. Condensed Matter Physics 1997 No 11 (117-129) 
function. The approaches based on the coherent potential approximation provide effective tools for the calculation of a polaron self-energy function for a wide class of models which allow us consequently (nonperturbatively) to account multiphonon scattering. The attempts to construct such an algorithm in a case of a linear electron(exciton)-phonon coupling were made before. In [4] the self- energy function was expanded into integral chain fraction with the use of a diagram technique. In [5] the expansion of Green's function into continued fraction was performed by means of the Haydock method. It is known that the coherent potential approximation (CPA) is the most sophisticated virtual-crystal approximation scheme for disordered electronic systems [6-8]. The first attempt to use the notion of the coherent potential for periodic solids in which the role of disorder is played by other elemental excitations which interact with the electron, appeared probably in the $s-d$ model of magnetic semiconductors $[9,10]$. Almost simultaneously with the coherent potential method for magnetic semiconductors, the so-called (local) Dynamical Coherent Potential Approximation (DCPA) was suggested by Sumi [11-13]. This approximation can be applied to a system in which only an Einstein phonon spectrum and a site diagonal electron (exciton)-phonon coupling are assumed. For a system with a non-diagonal electron(exciton)-phonon coupling the DCPA is completely inadequate because of the important role of correlated scattering among different sites. Another difficulty in the use of the DCPA is that the resulting condition for the coherent potential in [11-13] requires a numerical solution of a set of functional equations. Lately several attempts have been made to improve the (local) DCPA [14-18]. In [14] the dynamical CPA was reformulated in terms of the so-called locators that allow one to simplify the resulting set of equations for the coherent potential. S.Abe extended the (local) DCPA to two-particle Green's functions [15,16], and reformulated this approximation for a model of a Frenkel exciton coupled to dispersive phonons $[17,18]$. The (local) DCPA was employed to calculate energy spectra [11], absorption and emission spectra [12,17], resonance Raman spectra, and time-resolved spectra [16] in exciton-phonon systems. It has been successfully used to explain experiments of absorption spectra in molecular crystals $[19,20]$.

The present paper is devoted solely to the theoretical formulation of the NDCPA, whereas the results of actual calculations using this formulation will be presented in a separate paper. The dynamical CPA is an one-site approximation in which variation of a site local environment (due to the presence, for example, of phonons with dispersion) is ignored. It is known from the coherent potential theory for disordered solids [21] that one can account in some length the variation of a site local environment through the introduction of a nonlocal coherent potential which depends on the difference between site coordinates. The Fourier transform of the coherent potential is a function of a quasiimpulse vector. It is clear that the introduction of the nonlocal coherent potential in the above way is another level of approximations because in that case the system is implied to have a translational symmetry. For the dynamical disorder which is brought about by phonons the introduction of the nonlocal coherent potential is natural and one does not make any approximation at this. The proposed nonlocal dynamical coherent potential approximation (NDCPA) follows this way to improve the dynamical CPA. In fact, NDCPA can be used for the calculation of a single-particle Green's function of a model of the Hamiltonian with the electron(exciton)-phonon coupling of any structure. This approach also has technical preferences: at zero temperature the algorithm for calculating the coherent potential (as a function of the electron(exciton) impulse vector 
and energy) is reduced to a set of recurrent linear equations. The algorithm is greatly simplified if only inelastic scattering of electron(exciton) by phonons are taken into account. This is possible if the polaron spectrum is shifted in the energy region where processes of elastic scattering can be neglected. In particular, this situation may be realized in the case of an optical phonon spectrum and a narrow electron(exciton) bandwidth (antiadiabatic limit). This problem will be discussed in more details in Sec.3. In the antiadiabatic limit and Einstein phonon spectrum a Green's function can be obtained analytically for the case of a model with a linear and electron(exciton) site diagonal electron(exciton)-phonon interaction. If the energy gain of exciton localization is much larger than the electron(exciton) bandwidth the algorithm is allowed for the calculation of the lowest (localized) electron(exciton) state at the arbitrary ratio of electron(exciton) and phonon bandwidths. In the antiadiabatic limit and purely local electron (exciton)-phonon interaction it is also possible to obtain a simple recurrent algorithm in which elastic scattering of electron(exciton) in phonon field are accounted.

The paper is organized as follows: in the next section the model is introduced. In section 3 the nonlocal dynamical CPA at zero temperature is presented. The NDCPA is applied in the antiadiabatic limit for two cases. The first case relates to the polaron energies at which elastic scattering can be neglected. In the second one the elastic n-phonon short-range scattering correction is obtained.

\section{Model Hamiltonian}

In order to demonstrate the NDCPA a model of a system of excitons strongly coupled with phonons in a crystal with one molecule per unit cell is chosen. This model is called here a molecular crystal model. The Hamiltonian of this system is written as

$$
H=\sum_{k} E_{k} a_{k}^{+} a_{k}+\sum_{q} \omega(q)\left(b_{q}^{+} b_{q}+\frac{1}{2}\right)+\frac{1}{N^{1 / 2}} \sum_{n, q} a_{n}^{+} a_{n} \chi(q) \mathrm{e}^{i q n}\left(b_{q}^{+}+b_{-q}\right) .
$$

Here $a_{k}\left(a_{k}^{+}\right)$is the annihilation (creation) operator of an exciton with momentum $k$ and energy $E_{k}$, operator $a_{n}\left(a_{n}^{+}\right)$annihilates (creates) an exciton at the $n$th site, $b_{q}\left(b_{-q}^{+}\right)$is the annihilation (creation) operator of a phonon with momentum $q$ and energy $\omega(q), \chi(q)$ is the exciton-phonon coupling function, $N$ is the total amount of crystal molecules. The exciton energy is $E_{k}=\epsilon_{o}+t_{k}$, where $\epsilon_{0}$ is the change of energy of the crystal molecule under excitation, and $t_{k}$ is the Fourier transform of the energy transfer matrix elements.

This model can be employed to describe an exciton interacting with molecular as well as with lattice vibrations. The coupling function $\chi$ is $q$-independent if the exciton-phonon interaction is taken to be site-diagonal and purely local in the exciton and phonon coordinates. This case is realized, for example, for the exciton interacting with molecular vibrations. The $q$-dependent coupling function represents the interaction of the exciton with lattice phonons that arise due to the changing of van der Waals and Coulomb forces between molecules under lattice vibrations. The accounting of the resonant interaction is resulted in $k$-dependent terms in the coupling function. In general, the magnitude of $k$-dependent and independent terms in the coupling function varies from one material to another. For a material 
in which the interaction of the exciton with local environment is strong the exciton-phonon interaction operator can be taken as in (1) with the appropriately chosen coupling function $\chi(q)$.

Hamiltonians equivalent to (1) have been used by many authors for the consideration of a wide variety of problems which relate to the interaction of electrons or excitons with local environment in solids [22-25]. The model with Hamiltonian in which the terms describing the interaction between excitons or electrons are included is also allowed for the use of NDCPA. For example, the Hamiltonian (1) with the electron-electron interaction terms accounted becomes equivalent to the Hamiltonians (for instance, of the Holstein type) of some theories of superconductivity [26-28].

\section{Nonlocal Dynamical CPA}

Let us introduce the coherent potential $v_{k}(E)$ which is thought to be dependent on energy $E$ and exciton impulse $k$. The coherent potential is translational invariant in a site representation. The Hamiltonian (1) is transformed with the coherent potential taken into account as

$$
H=H^{e f f}+H^{\prime},
$$

with

$$
\begin{aligned}
H^{e f f} & =\sum_{k}\left(E_{k}+v_{k}\right) a_{k}^{+} a_{k}+\sum_{q} \omega(q)\left(b_{q}^{+} b_{q}+\frac{1}{2}\right), \\
H^{\prime} & =\sum_{n} a_{n}^{+} a_{n} \varphi_{n}-\sum_{n, m} v_{n, m} a_{n}^{+} a_{m} .
\end{aligned}
$$

In $H^{\prime}$ a site representation is used and

$$
\varphi_{n}=\sum_{m} g_{n, m}\left(b_{m}^{+}+b_{m}\right),
$$

where

$$
b_{m}=\frac{1}{N^{1 / 2}} \sum_{q} b_{q} \mathrm{e}^{i q m}
$$

is the annihilation operator of a phonon associated with the $m$ th lattice site, and

$$
\begin{aligned}
g_{n, m} & =\frac{1}{N} \sum_{q} \chi(q) \mathrm{e}^{i q(n-m)}, \\
v_{n, m} & =\frac{1}{N} \sum_{k} v_{k} \mathrm{e}^{i k(n-m)} .
\end{aligned}
$$

In further manipulations a site representation will be used for convenience. The Fourier transform with respect to time of a single-exciton delayed Green's function $G^{e f f}(t)$ of the system under the Hamiltonian $H^{e f f}$ in a 
site representation for exciton freedom degrees and Fock's representation for phonon freedom degrees is written as

$$
\begin{array}{r}
G_{m l}^{e f f}(z)=\frac{1}{N} \sum_{k} \sum_{s=0}^{\infty} \frac{1}{s !} \frac{1}{N^{s}} \sum_{\substack{q_{1}, \ldots, q_{s} \\
i_{1}, \ldots, i_{s} \\
j_{1}, \ldots, j_{s}}} \frac{\mathrm{e}^{i k(m-l)} \mathrm{e}^{i q_{1}\left(i_{1}-j_{1}\right)+\cdots+i q_{s}\left(i_{s}-j_{s}\right)}}{z-E_{k}-v_{k}(z)-\omega\left(q_{1}\right)-\cdots-\omega\left(q_{s}\right)} \times \\
\left|i_{1}, \ldots, i_{s}\right\rangle\left\langle j_{1}, \ldots, j_{s}\right| .
\end{array}
$$

Here

$$
\left|i_{1}, \ldots, i_{m}\right\rangle=b_{i_{1}}^{+} \cdots b_{i_{m}}^{+}|0\rangle,
$$

and $z$ denotes complex energy $z=E+i \gamma$ with exciton decay rate $\gamma$ which is assumed to be independent of energy and momentum. It is assumed in the expressions below that summations are performed over indexes $s, s_{i}, i=1,2, \ldots$ Expanding the Green's function $G_{m l}(z)$ of the exciton under Hamiltonian $H$ in powers of propagator $G_{m l}^{e f f}(z)$ we get

$$
\begin{aligned}
G_{m l}(z) & =\left[z-H_{e f f}-(\hat{\varphi}-\hat{v}(z))\right]_{m l}^{-1}= \\
& =G_{m l}^{e f f}(z)+G_{m s}^{e f f}(z)(\hat{\varphi}-\hat{v}(z))_{s s_{1}} G_{s_{1} l}^{e f f}(z)+\cdots .
\end{aligned}
$$

Here $\hat{\varphi}$ and $\hat{v}(z)$ are operator matrixes with elements

$$
\varphi_{m n}=\delta_{m n} \varphi_{n}
$$

and $v_{m n}(z)$ respectively.

We will restrict ourselves to the case of a system at zero temperature. This is not relevant from the point of view of methodology. The case of nonzero temperatures may be considered in a completely analogous fashion. At zero temperature the coherent potential is chosen such that $G_{m l}(z)$ and $G_{m l}^{e f f}(z)$ averaged over phonon vacuum are equal

$$
\left\langle 0\left|G_{m l}(z)\right| 0\right\rangle=\left\langle 0\left|G_{m l}^{e f f}(z)\right| 0\right\rangle .
$$

For the case of nonzero temperatures the vacuum averages in equation (7) should be replaced by thermal averages over phonon populations. Using (7) and (5) we get that scattering of the exciton in the effective medium by perturbation $(\hat{\varphi}-\hat{v}(z))$ is described by the following self-consistent condition

$$
\begin{aligned}
\left\langle 0\left|(\hat{\varphi}-\hat{v}(z))_{m l}+(\hat{\varphi}-\hat{v}(z))_{m s} G_{s s_{1}}^{e f f}(z)(\hat{\varphi}-\hat{v}(z))_{s_{1} l}+\cdots\right| 0\right\rangle & = \\
=\left\langle 0\left|T_{m l}\right| 0\right\rangle & =0 .
\end{aligned}
$$

Performing summation in (8) we obtain the $t$-matrix operator $\hat{T}$. Once $\hat{T}$ is found equation (8) is written as

$$
\left\langle 0\left|\left[\hat{I}-(\hat{\varphi}-\hat{v}(z)) \hat{G}^{e f f}(z)\right]^{-1}(\hat{\varphi}-\hat{v}(z))\right| 0\right\rangle=0 .
$$

$\hat{I}$ is a unity matrix. The Green's function $\hat{F}(z)$ of the exciton under the Hamiltonian (1) in which the exciton-phonon coupling terms are excluded is given by the expression

$$
\hat{F}^{-1}(z)=\hat{G}^{e f f-1}(z)+\hat{v}(z) .
$$


With the use of the operator $\hat{F}(z)$ equation (9) then is rewritten as

$$
\left\langle 0\left|[\hat{I}-\hat{\varphi} \hat{F}(z)]^{-1} \hat{\varphi}\right| 0\right\rangle=\left\langle 0\left|[\hat{I}-\hat{\varphi} \hat{F}(z)]^{-1}\right| 0\right\rangle \hat{v}(z) .
$$

It is an operator (or matrix) equation for the coherent potential $\hat{v}(z)$. The formal expression for $\hat{v}(z)$ can instantly be written. The exciton polaron is determined by the Green's function for the exciton in the effective medium defined by the coherent potential

$$
\left\langle 0\left|G_{m l}^{e f f}(z)\right| 0\right\rangle=\frac{1}{N} \sum_{k} \frac{\mathrm{e}^{i k(m-l)}}{z-E_{k}-v_{k}(z)} .
$$

The question is how to calculate the vacuum averages in equation (11). Below a set of equations that allow us to evaluate these averages is derived.

Let $\tilde{i}_{n}, \tilde{i}_{n}+i_{k}$, and $\tilde{i}_{n}-i_{k}$ represent sets of sites $\left(i_{1}, \ldots, i_{n}\right),\left(i_{1}, \ldots, i_{n}, i_{k}\right)$, and $\left(i_{1}, \ldots, i_{k-1}, i_{k+1}, \ldots, i_{n}\right)$ respectively. The $(0)$ and $\left(i_{1}\right)$ represent respectively empty and one-site sets. In the expressions below we do not always indicate the dependence of the operators $\hat{F}(z)$ and $\hat{v}(z)$ on energy $z$. All the calculations are performed with $\hat{F}(z), \hat{v}(z)$ taken at the same energy. Defining

$$
a_{m l}^{\tilde{i}_{n}}=\left\langle 0\left|[\hat{I}-\hat{\varphi} \hat{F}]_{m l}^{-1}\right| i_{1}, \ldots, i_{n}\right\rangle
$$

and using (11), (6), and (3) the coherent potential $v_{m l}$ is expressed as

$$
v_{m l}=\left[a^{(0)}\right]_{m s}^{-1} a_{s l}^{\left(s_{1}\right)} g_{s_{1} l} .
$$

Employing the obvious operator identity

$$
[\hat{I}-\hat{\varphi} \hat{F}]^{-1}=\hat{I}+[\hat{I}-\hat{\varphi} \hat{F}]^{-1} \hat{\varphi} \hat{F},
$$

and definition (13) we get that the matrix elements $a_{m l}^{\tilde{i}_{n}}$ are defined by the following set of equations

$$
\begin{gathered}
a_{m l}^{(0)}=\delta_{m l}+a_{m s}^{\left(s_{1}\right)} g_{s s_{1}} F_{s l}^{(0),(0)} \\
\vdots \\
a_{m l}^{\tilde{i}_{n}}=a_{m s}^{\tilde{s}_{n}+s_{1}}\left(n_{\tilde{s}_{n}, s_{1}}+1\right)^{1 / 2} g_{s s_{1}} F_{s l}^{\tilde{s}_{n}, \tilde{i}_{n}}+a_{m s}^{\tilde{s}_{n}-s_{1}}\left(n_{\tilde{s}_{n}, s_{1}}\right)^{1 / 2} g_{s s_{1}} F_{s l}^{\tilde{s}_{n}, \tilde{i}_{n}}
\end{gathered}
$$

Here

$$
F_{m l}^{\tilde{i}_{n}, \tilde{j}_{n}}(z)=\frac{1}{N^{n+1}} \sum_{\substack{k \\ q_{1}, \ldots, q_{n}}} \frac{\mathrm{e}^{i k(m-l)} \mathrm{e}^{i q_{1}\left(i_{1}-j_{1}\right)+\cdots+i q_{n}\left(i_{n}-j_{n}\right)}}{z-E_{k}-\omega\left(q_{1}\right)-\cdots-\omega\left(q_{n}\right)}
$$

is a $n$-phonon matrix element of the operator $\hat{F}$ and $n_{\tilde{i}_{n}, i_{k}}=\left(\delta_{i_{1}, i_{k}}+\cdots+\right.$ $\left.\delta_{i_{n}, i_{k}}\right)$. (16) is a set of recurrent linear equations from which $a_{m l}^{(0)}$ and $a_{m l}^{\left(i_{1}\right)}$ can be determined. Inserting then $a_{m l}^{(0)}$ and $a_{m l}^{\left(i_{1}\right)}$ in equation (14) one finally finds the coherent potential. In system (16) only one equation - with $a_{p p}$ in the left-hand side, is inhomogeneous. Other equations are homogeneous 
and their form is independent of the index $m$. From this it can be easily shown that the matrix elements $a_{m l}^{(0)}$ and $a_{m l}^{\left(p_{1}\right)}$ are proportional

$$
a_{m l}^{\left(p_{1}\right)}=v^{\left(p_{1}-l\right)} a_{m l}^{(0)},
$$

with the coefficients of proportionality $v^{\left(p_{1}-l\right)}$. Inserting (18) in (14) we get that the coherent potential matrix $v_{m l}$ is actually diagonal with the diagonal elements

$$
v_{l l}=v^{\left(s_{1}-l\right)} g_{s_{1} l},
$$

and thus the coherent potential $v(z)=v_{l l}(z)$ is actually independent of the exciton impulse vector $k$. Therefore, to determine the coherent potential one needs to find from equations $(16)$ the values $a_{l l}^{(0)}$ and $a_{l l}^{\left(p_{1}\right)}$, and then calculate the values $v^{\left(p_{1}\right)}$ by the formula

$$
v^{\left(p_{1}\right)}=\frac{a_{l l}^{\left(p_{1}\right)}}{a_{l l}^{(0)}} .
$$

Inserting these values in (19) one finally obtains the coherent potential. With the use of equations (11), (15) one can easily obtain similar to (16) equations for the case of exciton-phonon coupling operator $\hat{\varphi}$ of a more complex (for instance, quadratic) with respect to phonon operators structure.

Equations (16) are greatly simplified if we make some assumptions which allow the exciton and phonon site nondiagonal matrix elements $F_{m l}^{\tilde{i}_{n}, \tilde{j}_{n}}$ to be neglected. In the case of an Einstein phonon spectrum these matrix elements are exactly zero and equations (16) contain only $F_{m l}^{\tilde{i}_{m}, \tilde{i}_{n}}$. For further reference the matrix elements of $\hat{F}$ which are site diagonal in exciton coordinates, and both in exciton and phonon coordinates are denoted respectively as

$$
\begin{aligned}
& F_{m l}^{(n)}(z)=\frac{1}{N^{n+1}} \sum_{\substack{k \\
q_{1}, \ldots, q_{n}}} \frac{\mathrm{e}^{i k(m-l)}}{z-E_{k}-\omega\left(q_{1}\right)-\cdots-\omega\left(q_{n}\right)}, \\
& F^{(n)}(z)=\frac{1}{N^{n+1}} \sum_{\substack{k \\
q_{1}, \ldots, q_{n}}} \frac{1}{z-E_{k}-\omega\left(q_{1}\right)-\cdots-\omega\left(q_{n}\right)} .
\end{aligned}
$$

The nondiagonal $F_{m l}^{\tilde{i}_{n}, \tilde{j}_{n}}$ can be neglected at the energies $E$ which satisfy the following condition

$$
\left|E-\epsilon_{0}-n \omega(0)\right| \gg B+n \Omega,
$$

where $B$ and $\Omega$ are exciton and phonon bandwidths respectively. The fact that this condition is unfulfilled for some value of energy $E$ means that for a polaron state with this energy elastic (with impulse transfer) $n$-phonon scattering of the exciton is relevant. We can account $F^{(n)}$ in equations (16) only if it is supposed that the polaron spectrum is located in the energy region where elastic scattering are irrelevant. This situation may be realized in the case of a low dispersion optical phonon spectrum $(\Omega \ll \omega(0))$, and a narrow exciton band $(B \ll \omega(0))$. As it will be seen, in this case the polaron spectrum is shifted by the energy gain of exciton localization. Due to this shifting the energies in the resulted polaron bands may obey 
condition (23). If condition (23) is incorrect for the polaron band with some $n_{0}$, then $n_{0}$-phonon elastic scattering of the exciton should be taken into account and, therefore, $F_{m l}^{\tilde{i}_{n},} \tilde{j}_{n_{0}}$ should be accounted in equations (16). In other words, in this case we should make elastic scattering corrections for the calculation of this polaron band. It is obviously that if the phonons are acoustical the elastic $n$-phonon scattering become relevant beginning with a sufficiently large $n$ for an arbitrary value of the polaron energy $E$ (see $(23))$.

Let us first consider a case of the polaron spectrum when condition (23) is fulfilled and thus the nondiagonal matrix elements of operator $\hat{F}$ can be dropped out. We denote the obtained coherent potential in which only inelastic scattering are accounted as $v_{\text {inel }}$. By the explicit calculations it is easy checked that

$$
\frac{a_{m l}^{\tilde{i}_{n}+p}}{a_{m l}^{\tilde{i}_{n}+p^{\prime}}}=\frac{g_{l p}}{g_{l p^{\prime}}} \frac{\left(n_{\tilde{i}_{n}, p^{\prime}}\right)^{1 / 2}}{\left(n_{\tilde{i}_{n}, p}\right)^{1 / 2}} .
$$

Therefore, equations (16) contain only $a_{m l}^{\tilde{p}_{n}}$. Here $\tilde{p}_{n}=(\underbrace{p, \ldots, p}_{n})$. It is easily seen that $a_{m l}^{\tilde{p}_{n}}=0$ when $m, l \neq p$. Finally we conclude that equations (16) actually form a three-diagonal set for the values $a_{p p}^{\tilde{p}_{n}}$. This enables us to express the coherent potential in the form of a continued fraction. In order to show this let us introduce $t^{(n)}$ written as

$$
t^{(n)}=\frac{a_{p p}^{\tilde{p}_{n}}}{a_{p p}^{\tilde{p}_{n}-1}} \frac{1}{n^{1 / 2}} .
$$

Then equations (16) combined with equations (14) and (24) yield

$$
\begin{gathered}
v_{\text {inel }}(z)=t^{(1)} \frac{G}{g_{00}} \\
\vdots \\
t^{(n)}=\frac{1}{\frac{1}{F^{(n)}(z)}-(n+1) t^{(n+1)} \frac{G}{g_{00}}} \\
\vdots
\end{gathered}
$$

where $g_{00}=g_{p p}$ and

$$
G=\sum_{s} g(s)^{2}=\frac{1}{N} \sum_{q}|\chi(q)|^{2} .
$$

From equations $(26)$ we get the coherent potential $v_{\text {inel }}$ in the form

$$
v_{\text {inel }}(z)=\frac{G}{\frac{1}{F^{(1)}(z)}-\frac{2 G}{\frac{1}{F^{(2)}(z)}-\frac{3 G}{\ddots}} .}
$$


The energy spectrum of the exciton polaron is obtained as a function of $k$ by the solution

$$
z-E_{k}-v(z)=0 .
$$

Equation (28) with $v(z)=v_{\text {inel }}(z)$ can be further simplified if one assumes an Einstein phonon spectrum $\left(\omega(q)=\omega_{0}\right)$ and neglects in $F^{(n)}$ the elements of an energy transfer matrix (in this case $F^{(n)-1}(z)=z-\epsilon_{0}-n \omega_{0}$ ). Then, with the use of formula [29]

$$
\sum_{n=0}^{\infty} \frac{a^{n} / n !}{z+a-n} \mathrm{e}^{-a}=\frac{1}{z-\frac{a}{z-1-\frac{2 a}{z-2-\frac{3 a}{\ddots}}}}
$$

for complex $z$ with a nonvanishing imaginary part equation (28) is written as

$$
t_{k}-\frac{1}{\sum_{n=0}^{\infty}\left(\frac{1}{z-\epsilon_{0}+S \omega_{0}-n \omega_{0}}\right) \frac{S^{n}}{n !} \mathrm{e}^{-S}}=0,
$$

where $S=G / \omega_{0}^{2}$. For a limiting case $t_{k}=0(B=0)$ when the exciton energy spectrum converges to $\epsilon_{0}$ which may correspond to a highly localized exciton or an exciton trapped on impurity we get from (12), (28), and (29) the exciton Green's function in the form

$$
G(z)=\sum_{n=0}^{\infty}\left(\frac{1}{z-\epsilon_{0}+S \omega_{0}-n \omega_{0}}\right) \frac{S^{n}}{n !} \mathrm{e}^{-S} .
$$

This result can be easily obtained by usual methods [30,31]. Thus, we see that the present approximation becomes valid for $B=0$. On the whole, formula (29) accounts the configuration mixing among polaron states with a different number of phonons. Let us note that in the case $S>1$ several terms of the sum in the denominator of equation (29) may make the contribution of the same order to the energies of a polaron band (i.e. excitonphonon scattering with a different number of phonons should be accounted in the calculations of this polaron band), whereas for $S \ll 1$ there is only one leading term, that is in accordance with [4]. It is seen that the polaron spectrum calculated with the use of equation (29) consists of equally spaced bands which are shifted to lower energies by $S \omega_{0}$ and have a renormalized dispersion law comparatively with a dispersion law of a free exciton. To see the latter let us suppose that for energies in the $n_{0}$-th polaron band we have only one leading term of the sum in (29) which corresponds to $n_{0}-$ phonon scattering. Then the dispersion law in the $n_{0}-$ th polaron band may be roughly described by the formula

$$
E=\epsilon_{0}-S \omega_{0}+n_{0} \omega_{0}+\frac{S^{n_{0}}}{n_{0} !} \mathrm{e}^{-S} t_{k}
$$

Such a renormalization can be obtained within the framework of the small polaron theory [3]. $S \omega_{0}$ is the energy gain of exciton localization. Let us note that condition (23) and, therefore, equation (29) are correct for $S \gg B / \omega_{0}$ 
and for arbitrary $B / \omega_{0}$ for the lowest energy of the exciton polaron. So equation (29) is allowed to evaluate the energy of a self-trapped exciton when the energy of a vibrational or lattice relaxation is much larger than the exciton bandwidth.

It is possible to make elastic scattering corrections to algorithm (27) in the case of an Einstein phonon spectrum and a purely site-diagonal excitonphonon coupling. If we calculate the energy of the polaron state at the value $E \approx n \omega_{0}$, we should account in equations (16) the matrix elements $F_{m l}^{(n)}$ only. In this case equations (16) are rewritten as

$$
\begin{gathered}
a_{m p}^{(0)}=\delta_{m p}+a_{m p}^{(p)} g_{00} F^{(1)} \\
\vdots \\
a_{m p}^{\tilde{p}_{n-1}}=a_{m p}^{\tilde{p}_{n}} F^{(n-1)}(n)^{1 / 2} g_{00}+a_{m p}^{\tilde{p}_{n-2}} F^{(n-1)}(n-1)^{1 / 2} g_{00} \\
a_{m l}^{\tilde{p}_{n}}=\sum_{s \neq p} a_{m s}^{\tilde{p}_{n}+s} F_{s l}^{(n)} g_{00}+a_{m p}^{\tilde{p}_{n+1}} F_{p l}^{(n)}(n+1)^{1 / 2} g_{00}+a_{m p}^{\tilde{p}_{n-1}} F_{p l}^{(n)} n^{1 / 2} g_{00} \\
a_{m l}^{\tilde{p}_{n}+l}=a_{m l}^{\tilde{p}_{n}+\tilde{l}_{2}} F^{\left(n \delta_{p l}+1\right)}\left(n \delta_{p l}+2\right)^{1 / 2} g_{00}+a_{m l}^{\tilde{p}_{n}} F^{\left(n \delta_{p l}+1\right)}\left(n \delta_{p l}+1\right)^{1 / 2} g_{00}
\end{gathered}
$$

Here $\tilde{p}_{n}+\tilde{l}_{k}=(\underbrace{p, \ldots, p}_{n}, \underbrace{l, \ldots, l}_{k})$. It is easily seen that in equations (32) $a_{m l}^{\tilde{p}_{n}+\tilde{l}_{k}}=0$ and $a_{m l}^{\tilde{p}_{r}}=0$ when $m \neq p$. Equations (32) with $a_{p l}^{\tilde{p}_{n}+\tilde{l}_{k}}, p \neq l$ in the left-hand side form a three-diagonal set of equations for $a_{p l}^{\tilde{p}_{n}+\tilde{l}_{k}}$ completely analogical with equations (26). Therefore, resolving this system we obtain

$$
\frac{a_{p l}^{\tilde{p}_{n}+l}}{a_{p l}^{\tilde{p}_{n}}}=v_{i n e l} \frac{1}{g_{00}} .
$$

Let us now introduce a matrix with the elements

$$
t_{p l}=\frac{a_{p l}^{\tilde{p}_{n}}}{a_{p p}^{\tilde{p}_{n}}}
$$

We will also use values (25). Using then equations (32) and (33) we obtain that the coherent potential $v_{e l}$ with the elastic scattering correction is defined by the following equations

$$
\begin{gathered}
v_{e l}(z)=t^{(1)} g_{00} \\
t^{(n-1)}=\frac{\vdots}{\frac{1}{F^{(n-1)}(z)}-n t^{(n)} g_{00}} \\
t_{p l}=t_{p s} F_{s l}^{(n)}(z) v_{\text {inel }}(z)- \\
-F_{p l}^{(n)}(z) v_{\text {inel }}(z)+t^{(n+1)} F_{p l}^{(n)}(z)(n+1) g_{00}+\frac{1}{t^{(n)}} F_{p l}^{(n)}(z) g_{00},
\end{gathered}
$$




$$
t^{(n+1)}=\frac{g_{00}}{\frac{1}{F^{(n+1)}(z)}-(n+2) t^{(n+2)} g_{00}}
$$

From equations (35) for $t_{p l}$ when $p \neq l$ we can find the Fourier transform $t(k)$ of $t_{p l}$ through $t^{(n)}$ and $t^{(n+1)}$ only. Then inserting the obtained $t(k)$ in equations (35) with $t_{p p}$ in a left-hand side we get the equation which contains $t^{(n)}$ and $t^{(n+1)}$ only from which we can represent $t^{(n)}$ through $t^{(n+1)}$

$$
t^{(n)}=\frac{g_{00}}{\left(\frac{1}{N} \sum_{k} \frac{1}{z-E_{k}-n \omega_{0}-v_{\text {inel }}(z)}\right)^{-1}+v_{\text {inel }}(z)-(n+1) t^{(n+1)} g_{00}} .
$$

Equations (35) where $t^{(n)}$ is calculated by formula (36) represent the recurrent algorithm for the determination of $v_{e l}$.

It is seen from the above mathematics that the changes in the model Hamiltonian (1) which do not involve exciton-phonon coupling terms, - for instance, including the exciton-exciton (electron-electron) interaction, lead only to the respective change of $F_{m l}^{\tilde{i}_{n}} \tilde{j}_{n}$ in equations (16).

\section{Conclusions}

The NDCPA seems to be a very reasonable way to treat the properties of both electrons and excitons interacting with phonons with a dispersion. In fact, the NDCPA can be applied to a system of the Hamiltonian with electron(exciton)-phonon coupling terms of an arbitrary structure. The NDCPA resulted in an algorithm which can be effectively treated numerically (for example, iteratively). The application of the NDCPA is not restricted to a case of zero temperature and may be spread out to the systems with other distributions (thermal or nonequilibrium) of phonon populations. For such systems the averages in equation (7) should be performed over respective distributions. The algorithm is greatly simplified in the antiadiabatic limit and at the polaron energies when the elastic exciton-phonon scattering are irrelevant. In this case, if the electron(exciton)-phonon coupling term is linear, the NDCPA algorithm is reduced to a three-diagonal linear set of equations and, therefore, the coherent potential can be obtained analytically. It should be noted that for electron(exciton)-phonon terms, including the phonon operator of $n$th order the coherent potential is represented by a $2 n+1$-diagonal linear set of equations and can easily be found numerically up to the high order of electron(exciton)-phonons scattering. It is also possible to obtain the $n$-phonon short-range scattering correction when the role of these scattering is not negligible in the formation of a polaron band.

\section{References}

[1] Abrikosov A.A., Gor'kov L.P., Dzyaloshynskii I.E. Methods of Quantum Field Theory in Statistical Mechanics. New York, Dover, 1975.

[2] Lee. T. D., Pines D. The motion of slow electrons in polar crystals. // Phys. Rev., 1952, vol. 88, No 4, p. 960-961. 
[3] Lang I.G., Firsov Yu.A. Kinetic theory of semiconductors with low mobility. // Zh. Eksp. Teor. Fiz., 1962, vol. 43, No 5, p. 1843-1860 (in Russian).

[4] Tkach N.V. Method of the system of exact equations for the irreducible self-energy of quasiparticles interacting with phonons. // Teor. Mat. Fiz., 1984, vol. 61, No 3, p. 400-407 (in Russian).

[5] Sherman A.V. Absorption spectrum. // Phys. Stat. Sol. (b), 1985, vol. 131, No 1, p. 225-223.

[6] Soven P. Coherent-potential model of substitutional disordered alloys. // Phys. Rev., 1967, vol. 156, No 3, p. 809-813.

[7] Taylor D.W. Vibrational properties of imperfect crystals with large defect concentrations. // Phys. Rev., 1967, vol. 156, No 3, p. 1017-1029.

[8] Velicky B., Kirkpatrick S., Enrenreich H. Single-site approximation in the electronic theory of simple binary alloys. // Phys. Rev., 1968, vol. 175, No 3, p. 747-766.

[9] Rangette A., Yanase A., Kübler J. CPA treatment of the $s-d$ model at the high temperatures. // Sol. State Comm., 1973, vol. 12, No 1, p. 171-174.

[10] Kubo K. Electronic states in magnetic semiconductors. - An extension of CPA to random spin systems -. // J. Phys. Soc. Jpn., 1974, vol. 36, No 1, p. 32-38.

[11] Sumi H. Exciton polarons of molecular crystal model. I. - Dynamical CPA -. // J. Phys. Soc. Jpn., 1974, vol. 36, No 3, p. 770-779.

[12] Sumi H. Exciton polarons of molecular crystal model. II. - Optical spectra -. // J. Phys. Soc. Jpn., 1975, vol. 38, No 3, p. 825-835.

[13] Sumi H. Exciton-lattice interaction and the line shape of exciton absorption in molecular crystals. // J. Chem. Phys., 1977, vol. 67, No 7, p. 2943-2954.

[14] Čhápek V., Špička V. Polarons by coherent potential method. // Czech. J. Phys., 1984, vol. 34, No 2, p. 115-129.

[15] Abe S. Dynamical coherent potential approximation for light scattering spectra in exciton-phonon systems. // J. Phys. Soc. Jpn., 1988, vol. 57, No 11, p. 4029-4035.

[16] Abe S. Resonance Raman spectra and time-resolved emission spectra in exciton-phonon systems: calculation in the dynamical coherent potential approximation. // J. Phys. Soc. Jpn., 1988, vol. 57, No 11, p. 4036-4047.

[17] Abe S. Dynamics and optical spectra of an exciton in a deformable lattice. // J. Lumin., 1990, vol. 45, No 1-6, p. 272-274.

[18] Abe S. Dynamics of self-trapping of hot excitons strongly coupled to phonons. // J. Phys. Soc. Jpn., 1990, vol. 59, No 4, p. 1496-1505.

[19] Tokura Y., Koda T. Experimental determination of the charge-transfer exciton band width in anthracene-PMDA crystal. // Solid State Commun., 1981, vol. 40, No 3, p. 299-301.

[20] Wada Y., Tokura Y., Koda T. Analysis of the quasimetallic reflection band by dynamical coherent potential approximation (DCPA). // J. Chem. Phys., 1987, vol. 86, No 5, p. 3009-3020.

[21] Berk N.F., Shazeer D.J., Tahir-Kheli R.A. T-matrix range-truncation scheme for disordered alloys: nearest-neighbour truncation on one-dimensional model. // Phys. Rev. B, 1973, vol. 8, No 6, p. 2496-2504.

[22] Rashba E.I.. Dynamical theory of vibronic spectra in molecular crystals. // Zh. Eksp. Teor. Fiz., 1968, vol. 54, No 2, p. 542-558 (in Russian).

[23] Sumi H., Toyozawa Y. Urbach-Martienssen rule and exciton trapped momentarily by lattice vibrations. // J. Phys. Soc. Jpn., 1972, vol. 31, No 2, p. 342-358.

[24] Venzl G., Fischer S.F. Theory of exciton-phonon coupling in one-dimensional molecular crystals: a variational treatment with delocalized solitary states. // Phys. Rev. B, 1985, vol. 32, No 10, p. 6437-6446.

[25] Singh J., Matsui A. Free, quasifree, momentarily trapped, and self-trapped exciton states in molecular crystals. // Phys. Rev. B, 1987, vol. 36, No 11, p. 6094-6098.

[26] Holstein T. Studies of polaron motion. Part I. The molecular crystal model. // Ann. Phys. (New York), 1959, vol. 8, No 3, p. 325-342. 
[27] Ranninger J. Spectral properties of small-polaron systems. // Phys. Rev. B, 1993, vol. 48, No 17, p. 13166-13169.

[28] Marsiglio F. Pairing and charge-density-wave correlations in the Holstein model at half-filling. // Phys. Rev. B, 1990, vol. 42, No 4, p. 2416-2424.

[29] Gautschi W. In: Handbook of Mathematical Functions, edited by Abramowitz M. and Stegun I.A. New York, Dover, 1964, p. 295.

[30] Davydov A.S. Theory of molecular excitons. New York, Plenum Press, 1971.

[31] Davydov A.S. Theory of Solid State. Moscow, Nauka, 1976 (in Russian).

\section{ПОЛЯРОН У НАБЛИЖЕННІ НЕЛОКАЛЬНОГО ДИНАМІЧНОГО КОГЕРЕНТНОГО ПОТЕНЦІАЛУ}

\section{С.В.Ізвєков}

Сформульовано наближення нелокального динамічного когерентного потенціалу (ННДКП), яке дає змогу обчислити одноелектронну функцію Гріна полярона у випадку електрона(екситона), який взаємодіє з дисперсійними фононами. Запропоноване наближення є розширенням наближення динамічного когерентного потенціалу. ННДКП є ефективним засобом для обчислення наближеної функції Гріна для електрон(екситон)фононної системи у випадку сильного зв'язку та оптичного фононного спектру. Члени в модельному гамільтоніані, які описують електрон(екситон)-фононну взаємодію, можуть бути довільного степеня відносно фононних операторів народження та знищення. Отримано систему рекуррентних рівнянь у випадку системи при нульовій температурі, з якої можна знайти когерентний потенціал як функцію енергії $E$. Отримано простий алгоритм для розрахунку поляронного спектру у випадку лінійної електрон(екситон)-фононної взаємодії та в антиадіабатичному наближенні. 